\title{
Estimated respiratory arousal threshold in patients with rapid eye movement obstructive sleep apnea
}

\author{
Tetsuro Hoshino ${ }^{1}$ (D) $\cdot$ Ryujiro Sasanabe $^{1} \cdot$ Kenta Murotani $^{2} \cdot$ Reiko Hori $^{1} \cdot$ Mamiko Mano $^{1} \cdot$ Atsuhiko Nomura $^{1}$. \\ Noriyuki Konishi ${ }^{1} \cdot$ Masayo Baku ${ }^{1} \cdot$ Yoshitomo Nishio $^{3} \cdot$ Chihiro Kato $^{1} \cdot$ Wojciech Kuczynski ${ }^{4} \cdot$ Toshiaki Shiomi $^{1}$
}

Received: 21 October 2020 / Revised: 4 May 2021 / Accepted: 5 May 2021 / Published online: 17 May 2021

(c) The Author(s) 2021

\begin{abstract}
Purpose Rapid eye movement (REM) obstructive sleep apnea (OSA) is a prevalent clinical phenotype. However, the literature focusing on the pathophysiology of REM OSA is limited. This study compared the proportion of individuals with a low respiratory arousal threshold between patients with REM and non-REM OSA.

Methods REM OSA was defined as having an apnea-hypopnea index (AHI) $\geq 5$ and AHI during REM (AHI-REM)/AHI during NREM (AHI-NREM) $\geq 2$. REM OSA was sub-divided into REM-predominant OSA and REM-isolated OSA. REMpredominant OSA was defined as satisfying the definition of REM OSA and having an AHI-NREM $\geq 5$. REM-isolated OSA was defined as satisfying the definition of REM OSA and having an AHI-NREM $<5$. Patients with an AHI-REM/AHINREM $<2$ were defined as having non-REM OSA. A low respiratory arousal threshold was defined as having 2 or more of the following conditions: $\mathrm{AHI}<30$ events/h, proportion of hypopnea $>58.3 \%$, and nadir $\mathrm{SpO}_{2}>82.5 \%$.

Results The proportions of individuals with low respiratory arousal thresholds among individuals with REM-predominant OSA and REM-isolated OSA were significantly higher (77.2\% and $93.7 \%$, respectively) than that of patients with non-REM OSA (48.6\%). This was also true when the analysis was performed according to sex.

Conclusion These results indicate that a low respiratory arousal threshold might be an important endotype that contributes to the pathogenesis of REM OSA, especially in REM-isolated OSA.
\end{abstract}

Keywords Sleep apnea $\cdot$ REM obstructive sleep apnea $\cdot$ Phenotype $\cdot$ Insomnia $\cdot$ Respiratory arousal threshold

\section{Introduction}

Obstructive sleep apnea (OSA) is a heterogeneous disorder with both upper airway anatomical vulnerability and nonanatomical traits-including impairment in pharyngeal

Tetsuro Hoshino

hoshino.tetsurou.299@mail.aichi-med-u.ac.jp

1 Department of Sleep Medicine and Sleep Disorder Center, Aichi Medical University Hospital, 1-1 Nagakute, Aichi 4801195, Japan

2 Biostatistics Center, Graduate School of Medicine, Kurume University, 67 Asahimachi, Kurume, Fukuoka 8300011, Japan

3 Department of Oral and Maxillofacial Surgery, Aichi Medical University Hospital, 1-1 Nagakute, Aichi 4801195, Japan

4 Department of Sleep Medicine and Metabolic Disorders, Medical University of Lodz, 90-001 Lodz, Poland dilator muscle control and function to airway narrowing, respiratory control instability, and low arousal threshold [1]. Identifying the pathophysiological heterogeneity of OSA is essential for precision medicine.

Recent epidemiological studies have showed that rapid eye movement (REM) OSA is a highly prevalent clinical phenotype that affects $12.2-24.6 \%$ of patients with OSA and is characterized by apnea and hypopnea events that predominantly or exclusively occur during REM sleep [2]. Although personalized management strategies for OSA have been developed-including anatomical and non-anatomical approaches-few studies address the pathogenesis of REM OSA. This complicates efforts to describe the best treatment method for this entity [3]. Furthermore, REM OSA appears independently associated with adverse cardiovascular, metabolic, and neurocognitive outcomes [4]. Therefore, it is important to elucidate the pathophysiology underlying REM OSA. 
Recently, two studies have indicated that REM OSA was associated with depression and insomnia. The authors mentioned that a low respiratory arousal threshold might be an important endotype that contributes to the pathogenesis of REM OSA [5, 6]. Termination of respiratory events is associated with cortical arousal; therefore, respiratory arousal has been considered to be a potentially lifesaving event that prevents asphyxia during sleep [7]. Alternatively, low respiratory arousal thresholds contribute to prevent deeper stages of sleep associated with stable breathing, ventilatory instability, and increase cortical arousal and sleep fragmentation even in cases of mild upper airway narrowing. Repetitive arousals prevent the accumulation of chemical stimuli required to activate the upper airway dilator muscles [8]. Therefore, a low respiratory arousal threshold is an important endotype that contributes to the pathogenesis of OSA.

Currently, invasive procedures using epiglottic or esophageal pressure catheters must identify the respiratory arousal threshold [9]. However, Edwards et al. recently developed a clinical screening tool that identifies patients with OSA having a low respiratory arousal threshold based on three variables obtained from nocturnal polysomnography (PSG). This method correctly predicted a low respiratory arousal threshold in $84 \%$ of patients [9]. We used this clinical screening tool to identify individuals with a low respiratory arousal threshold from two groups of patients: those with REM OSA and those with non-REM OSA.

\section{Methods}

\section{Study population}

This single-center retrospective observational study examined 2531 patients who underwent PSG at the Department of Sleep Medicine of the Aichi Medical University Hospital from May 2013 to July 2020. A total of 659 patients were excluded: 166 patients aged $<18$ years, 5 patients who slept for $<2$ h during nocturnal PSG, 76 patients who were in REM sleep for $<10$ min, 401 patients with an apnea-hypopnea index (AHI) of $<5,10$ patients with chronic obstructive pulmonary disease (COPD), and 1 patient who was receiving oxygen therapy. Ultimately, 1872 adult patients with OSA were enrolled in the study (Fig. 1). At the time of their first visits to our department, the patients were required to complete the Pittsburgh Sleep Quality Index (PSQI) and Epworth Sleepiness Scale (ESS) questionnaires.

\section{Polysomnography}

Nocturnal PSG was performed using the SOMNOscreen plus PSG system (SOMNOmedics, Randersacker, Germany), Alice 5 or 6 System (Respironics Inc., Murrysville,

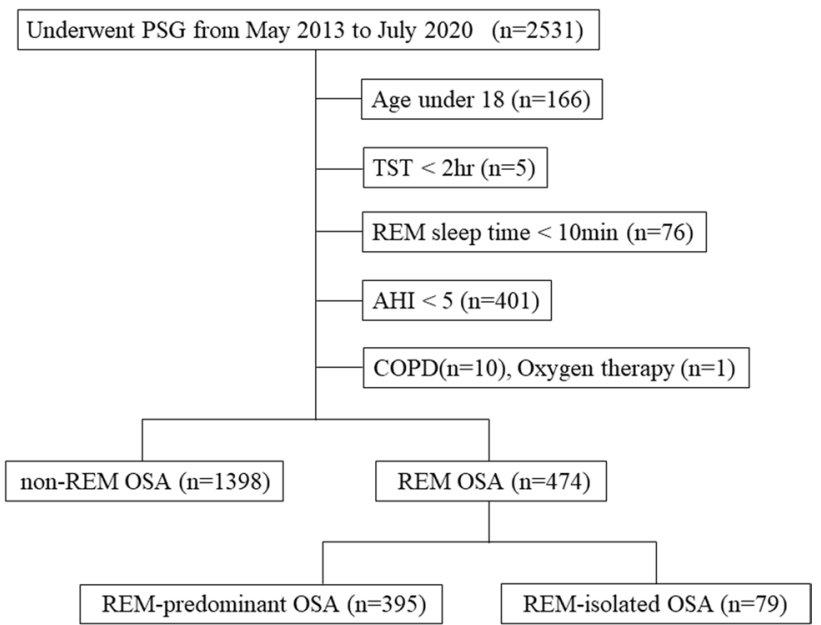

Fig. 1 Flow diagram of the study. AHI, apnea-hypopnea index; COPD, chronic obstructive pulmonary disease; OSA, obstructive sleep apnea; PSG, polysomnography; REM, rapid eye movement; TST, total sleep time

PA, USA), and PSG-1100 (Nihon Kohden Co., Tokyo, Japan). The following physiological parameters during sleep were simultaneously recorded: electro-encephalogram, electro-cardiogram, chin and anterior tibialis electro-myogram, bilateral electro-oculogram, nasal thermistor and prong pressure, respiratory inductance plethysmography bands on chest and abdomen, arterial oxygen saturation, body position, and snoring sound. All polysomnographic parameters were scored manually based on the American Academy of Sleep Medicine Manual for the Scoring of Sleep and Associated Events [10]. OSA was defined as having an AHI $\geq 5$ according to the International Classification of Sleep Disorders second edition criteria [11].

A low respiratory arousal threshold was defined as having two or more of the following conditions: $\mathrm{AHI}<30$ events/h, proportion of hypopnea $>58.3 \%$, and nadir $\mathrm{SpO}_{2}>82.5 \%$ [9].

\section{Definition of REM OSA, REM-predominant OSA, and REM-isolated OSA}

There was no uniform clinical definition for REM OSA at the time of this study. In reference to previous reports, REM OSA was defined as having an AHI $\geq 5$ and AHI-REM/AHINREM $\geq 2$ [3]. Moreover, we sub-classified REM OSA as follows:

- REM-isolated OSA: AHI $\geq 5$, AHI-REM/AHINREM $\geq 2$, and AHI-NREM $<5$.

- REM-predominant OSA: AHI $\geq 5$, AHI-REM/AHI$\mathrm{NREM} \geq 2$, and AHI-NREM $\geq 5$.

Patients with AHI-REM/AHI-NREM $<2$ were defined as having non-REM OSA. 


\section{Statistical analysis}

Continuous variables were expressed as medians and interquartile ranges. Comparison of polysomnographic and demographic features between patients with REM OSA and non-REM OSA was conducted using the Mann-Whitney $U$ test. Comparison between categorical variables was conducted using the Chi-square test. A multivariate logistic regression model was used to investigate the odds ratio with a 95\% confidence interval of patients with REM OSA for developing a respiratory arousal threshold score of $\geq 2$ after adjusting for medication, including hypnotics, antidepressants, and antipsychotics. Statistical analyses were performed using SAS 9.4 (SAS Institute Inc., Cary, NC, USA).

\section{Results}

Among the 1872 patients, 474 had REM OSA, and 1398 had non-REM OSA. Among patients with REM OSA, 395 patients satisfied the definition of REM-predominant OSA, and 79 patients satisfied the definition of REM-isolated OSA.

Table 1 shows the comparison of patients' characteristics and polysomnographic variables between REM OSA and non-REM OSA patients. Compared to patients with nonREM OSA, patients with REM OSA had a significantly higher PSQI and percentage of REM sleep/total sleep time (TST). In addition, the percentage of individuals satisfying each component of the respiratory arousal threshold score and those using hypnotic, antidepressant, and antipsychotic

Table 1 Comparison of patients' characteristics and polysomnographic variables between REM OSA and non-REM OSA patients

\begin{tabular}{|c|c|c|c|}
\hline & REM OSA $(n=474)$ & non-REM OSA $(n=1398)$ & $p$ Value \\
\hline Age (years) & $57(46,69)$ & $58(48,69)$ & 0.051 \\
\hline Male $(\%)$ & $275(58.0)$ & $1131(80.9)$ & $<.001^{*}$ \\
\hline BMI $\left(\mathrm{kg} / \mathrm{m}^{2}\right)$ & $25.1(22.5,28.8)$ & $25.0(22.6,28.4)$ & 0.861 \\
\hline ESS (points) & $7(4,11)$ & $8(5,12)$ & $0.027 *$ \\
\hline PSQI (points) & $7(5,12)$ & $6(4,9)$ & $<.001 *$ \\
\hline TST (min) & $455.0(433.9,487.1)$ & $460.5(439.0,488.0)$ & $0.030^{*}$ \\
\hline Percentage of REM sleep/TST (\%) & $16.9(12.1,21.3)$ & $15.8(11.7,20.3)$ & $0.009^{*}$ \\
\hline Percentage of N1 sleep/TST (\%) & $27.6(18.6,41.0)$ & $46.4(32.7,63.9)$ & $<.001 *$ \\
\hline Percentage of N2 sleep/TST (\%) & $52.7(41.2,61.9)$ & $36.0(20.4,48.1)$ & $<.001^{*}$ \\
\hline Percentage of N3 sleep/TST (\%) & $0(0,0.3)$ & $0(0,0.1)$ & 0.278 \\
\hline AHI (events/h) & $15.8(9.0,23.5)$ & $36.5(19.3,57.2)$ & $<.001^{*}$ \\
\hline AHI-REM (events/h) & $38.0(25.7,54.0)$ & $35.5(14.3,54.5)$ & $<.001 *$ \\
\hline AHI-NREM (events/h) & $10.9(5.9,18.0)$ & $36.8(19.8,57.8)$ & $<.001^{*}$ \\
\hline Nadir $\mathrm{SpO}_{2}(\%)$ & $84.0(79.0,88.0)$ & $82.0(74.0,87.0)$ & $<.001 *$ \\
\hline СТ90 (\%) & $0.6(0.1,2.1)$ & $1.6(0.1,8.1)$ & $<.001^{*}$ \\
\hline PLMI (events/h) & $0(0,10.1)$ & $0(0,7.0)$ & 0.052 \\
\hline ArI (events/h) & $23.5(17.3,31.7)$ & $39.0(27.5,55.7)$ & $<.001^{*}$ \\
\hline \multicolumn{4}{|l|}{ Components of arousal threshold score } \\
\hline AHI <30 events/h $(\%)$ & $419(88.4)$ & $552(39.5)$ & $<.001^{*}$ \\
\hline Proportion of hypopneas $>58.3$ ( $\%$ of all events) & $365(77.0)$ & $746(53.4)$ & $<.001^{*}$ \\
\hline Nadir $\mathrm{SpO} 2>82.5 \%(\%)$ & $274(57.8)$ & $658(47.1)$ & $<.001^{*}$ \\
\hline Proportion with arousal threshold score $\geq 2(\%)$ & $379(80.0)$ & $639(48.6)$ & $<.001 *$ \\
\hline Male $(\%)$ & $215(78.2)$ & $496(43.9)$ & $<.001 *$ \\
\hline Female $(\%)$ & $164(82.4)$ & $143(53.6)$ & $<.001 *$ \\
\hline \multicolumn{4}{|l|}{ Medication } \\
\hline Hypnotics (\%) & $164(34.6)$ & $267(19.1)$ & $<.001 *$ \\
\hline Antidepressants (\%) & $46(9.7)$ & $71(3.7)$ & $0.001 *$ \\
\hline Antipsychotics (\%) & $27(5.7)$ & $35(2.5)$ & $0.002 *$ \\
\hline
\end{tabular}

Continuous variables are expressed as medians and interquartile ranges (25th percentiles, 75 th percentiles). Categorical variables are expressed as numbers (percentages). ${ }^{*} p<0.05$

AHI apnea-hypopnea index, ArI arousal index, BMI body mass index, ESS Epworth Sleepiness Scale, N1 sleep stage 1, N2 sleep stage 2, N3 sleep stage 3, OSA obstructive sleep apnea, $P S Q I$ Pittsburgh Sleep Quality Index, $R E M$ rapid eye movement, $S p O_{2}$ peripheral capillary oxygen saturation, TST total sleep time 
medications were higher in the REM OSA compared to the non-REM OSA group.

Table 2 shows the comparison of patients' characteristics and polysomnographic variables between REM-predominant OSA and REM-isolated OSA patients. Compared to patients with REM- predominant OSA, patients with REM-isolated OSA showed a significantly higher PSQI and nadir $\mathrm{SpO}_{2}$. The percentage of individuals satisfying components of the respiratory arousal threshold score (including $\mathrm{AHI}<30$ events/h and nadir $\mathrm{SpO} 2>82.5 \%$ ) and those using hypnotics and antidepressants were higher in the REM-isolated OSA compared to the REM-predominant OSA group.

Significantly, more individuals with REM-predominant OSA and REM-isolated OSA demonstrated low respiratory arousal thresholds (77.2\% and $93.7 \%$, respectively) compared to patients with non-REM OSA (48.6\%). With respect to male patients, the proportions of individuals with low respiratory arousal thresholds among patients with REMpredominant OSA and REM-isolated OSA were significantly higher $(75.8 \%$ and $92.3 \%$, respectively) than that among patients with non-REM OSA (43.9\%) (Fig. 2). The same finding was true for females; the proportions of individuals with low respiratory arousal thresholds among patients with REM-predominant OSA and REM-isolated OSA were significantly higher (79.2\% and $95.0 \%$, respectively) than patients with non-REM OSA (53.6\%).

Table 3 shows the results of multivariate logistic regression analysis for the association between REM OSA and

Table 2 Comparison of patients' characteristics and polysomnographic variables between REM-predominant OSA and REM-isolated OSA patients

\begin{tabular}{|c|c|c|c|}
\hline & $\begin{array}{l}\text { REM-predominant OSA } \\
(n=395)\end{array}$ & REM-isolated OSA $(n=79)$ & $p$ Value \\
\hline Age (years) & $57(46,70)$ & $58(44,69)$ & 0.568 \\
\hline Male $(\%)$ & $236(59.7)$ & $39(49.4)$ & 0.104 \\
\hline BMI $\left(\mathrm{kg} / \mathrm{m}^{2}\right)$ & $25.6(22.8,29.3)$ & $23.4(21.5,25.8)$ & $<.001^{*}$ \\
\hline ESS (points) & $8(5,11)$ & $6(4,11)$ & 0.115 \\
\hline PSQI (points) & $7(5,11)$ & $9(6,13.5)$ & $0.030^{*}$ \\
\hline TST (min) & $455.0(433.0,487.0)$ & $457.0(441.0,493.5)$ & 0.536 \\
\hline Percentage of REM sleep/TST (\%) & $16.8(12.1,20.8)$ & $17.4(12.0,23.2)$ & 0.165 \\
\hline Percentage of N1 sleep/TST (\%) & $29.1(19.4,42.0)$ & $21.0(14.6,34.2)$ & $<.001^{*}$ \\
\hline Percentage of N2 sleep/TST (\%) & $51.9(39.4,61.3)$ & $57.2(49.1,65.7)$ & $<.001^{*}$ \\
\hline Percentage of N3 sleep/TST (\%) & $0(0,0.3)$ & $0(0,0.2)$ & 0.800 \\
\hline AHI (events/h) & $17.6(11.5,25.5)$ & $6.8(5.9,7.9)$ & $<.001^{*}$ \\
\hline AHI-REM (events/h) & $42.1(29.1,55.6)$ & $22.3(17.7,33.4)$ & $<.001^{*}$ \\
\hline AHI-NREM (events/h) & $12.9(8.1,19.4)$ & $3.8(2.9,4.2)$ & $<.001^{*}$ \\
\hline Nadir $\mathrm{SpO}_{2}(\%)$ & $83.0(78.0,87.0)$ & $88.0(84.0,91.0)$ & $<.001^{*}$ \\
\hline СТ90 (\%) & $0.8(0.1,2.3)$ & $0.1(0,0.5)$ & $<.001^{*}$ \\
\hline PLMI (events/h) & $0(0,10.4)$ & $0(0,9.2)$ & 0.973 \\
\hline ArI (events/h) & $24.5(18.1,32.1)$ & $18.8(13.7,25.1)$ & $<.001^{*}$ \\
\hline \multicolumn{4}{|l|}{ Components of arousal threshold score } \\
\hline $\mathrm{AHI}<30$ events/h $(\%)$ & $340(86.1)$ & $79(100)$ & $<.001^{*}$ \\
\hline Proportion of hypopneas $>58.3$ ( $\%$ of all events) & $306(77.5)$ & $59(74.7)$ & 0.660 \\
\hline Nadir SpO2>82.5\% (\%) & $208(52.7)$ & $66(83.5)$ & $<.001^{*}$ \\
\hline Proportion with arousal threshold score $\geq 2(\%)$ & $305(77.2)$ & $74(93.7)$ & $<.001^{*}$ \\
\hline Male $(\%)$ & $179(75.8)$ & $36(92.3)$ & $0.021 *$ \\
\hline Female $(\%)$ & $126(79.2)$ & $38(95.0)$ & $0.019^{*}$ \\
\hline \multicolumn{4}{|l|}{ Medication } \\
\hline Hypnotics (\%) & $126(31.9)$ & $38(48.1)$ & $0.007^{*}$ \\
\hline Antidepressants (\%) & $31(7.8)$ & $15(19.0)$ & $0.006^{*}$ \\
\hline Antipsychotics (\%) & $19(4.8)$ & $8(10.1)$ & 0.104 \\
\hline
\end{tabular}

Continuous variables are expressed as medians and interquartile ranges (25th percentiles, 75 th percentiles). Categorical variables are expressed as numbers (percentages). ${ }^{*} p<0.05$

AHI apnea-hypopnea index, ArI arousal index, BMI body mass index, ESS Epworth Sleepiness Scale, N1 sleep stage 1, N2 sleep stage 2, N3 sleep stage 3, OSA obstructive sleep apnea, $P S Q I$ Pittsburgh Sleep Quality Index, $R E M$ rapid eye movement, $\mathrm{SpO}_{2}$ peripheral capillary oxygen saturation, TST total sleep time 

als with a low arousal threshold score $\geq 2$. OSA, obstructive sleep apnea; REM, rapid eye movement
Fig. 2 Proportion of individu-

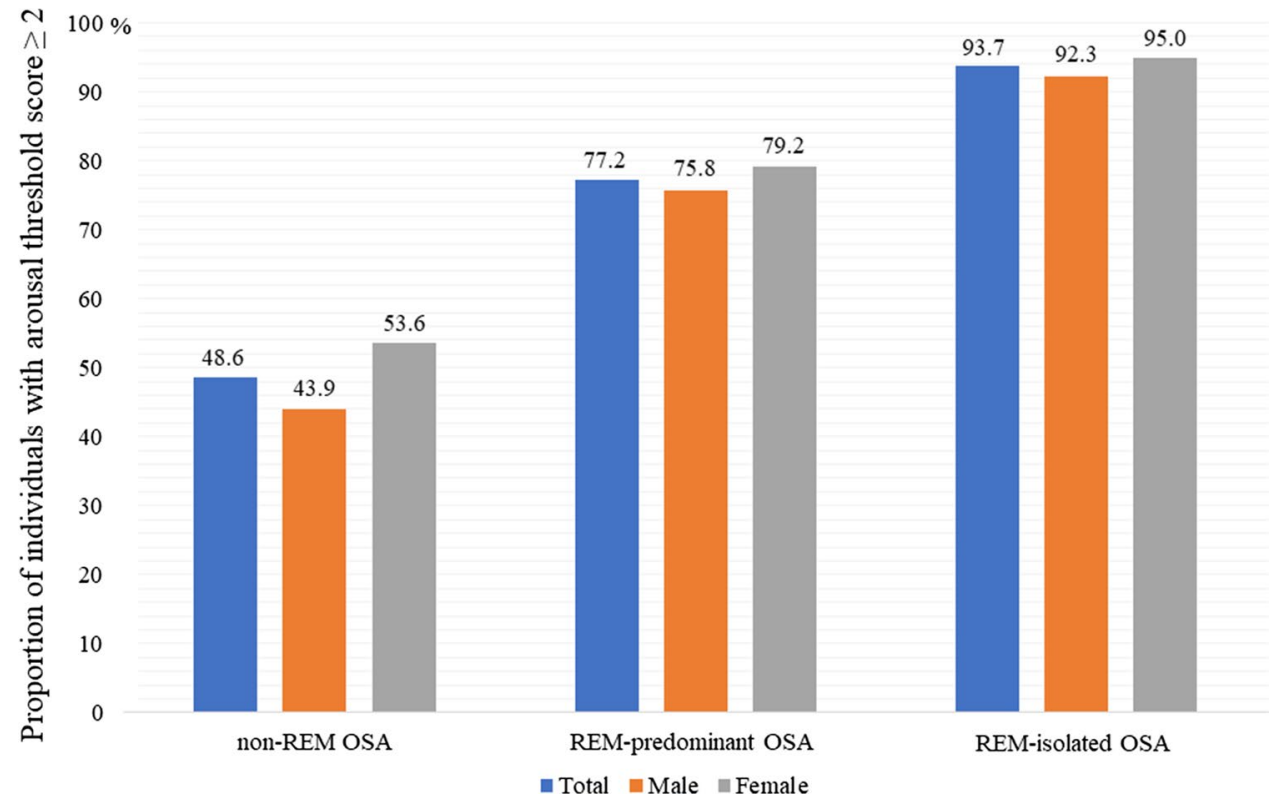

Table 3 Association between REM OSA and respiratory arousal threshold score $\geq 2$

\begin{tabular}{lllll}
\hline & Crude OR (95\% CI) & $p$ Value & Adjusted OR (95\% CI) & $p$ Value \\
\hline Non-REM OSA & 1 [Reference] & & 1 [Reference] & \\
REM-predominant OSA & $4.03(3.11-5.21)$ & $p<.001^{*}$ & $3.94(3.03-5.10)$ & $p<.001^{*}$ \\
REM-isolated OSA & $17.57(7.06-43.73)$ & $p<.001^{*}$ & $16.57(6.62-41.45)$ & $p<.001^{*}$ \\
\hline
\end{tabular}

$O S A$ obstructive sleep apnea, REM rapid eye movement, $O R$ odds ratio, $C I$ confidence interval. $* p<0.05$ having a respiratory arousal threshold score of $\geq 2$. In the unadjusted model, REM-predominant OSA (crude OR, 4.03; 95\% CI, 3.11-5.21; $p<0.001$ ) and REM-isolated OSA (crude OR, 17.57; 95\% CI, 7.06-43.73) were significantly associated with developing an arousal threshold score of $\geq 2$. In the model adjusted for medications including hypnotics, antidepressants, and antipsychotics, REM-predominant OSA (adjusted OR, 3.94; 95\% CI, 3.03-5.10; $p<0.001$ ) and REM-isolated OSA (adjusted OR, 16.57; 95\% CI, 6.62-41.45) were significantly associated with developing an arousal threshold score of $\geq 2$.

\section{Discussion}

In a previous study conducted at our institute, we found that insomnia, rather than daytime sleepiness, was an important complaint in patients with REM OSA [6]. Although the causal relationships have not been established, the previous study indicated a strong association between insomnia and low arousal thresholds [12]. Therefore, we conducted the current study focusing on the respiratory arousal threshold in patients with REM OSA.

All patients with OSA have some degree of upper airway vulnerability. A previous study found that $36 \%$ of patients with OSA had impairment in pharyngeal dilator muscle control and function to airway narrowing, 36\% had respiratory control instability, and 37\% had a low arousal threshold [1]. Our study showed that more than $90 \%$ of patients with REM-isolated OSA had a low respiratory arousal threshold. Zinchuk et al. found that non-obese patients with a low respiratory arousal threshold had worse adherence to CPAP therapy [13]. If, as we hypothesized, a low respiratory arousal threshold is a significant endotype of REM OSA, then this finding would be consistent with previous studies that reported low adherence to CPAP therapy among patients with REM OSA [14, 15]. Randomized controlled trials have successfully demonstrated that eszopiclone, zopiclone, and zolpidem increase respiratory arousal thresholds $[3,8]$. Therefore, the use of these drugs may improve CPAP therapy adherence in patients with REM OSA.

Patients with REM OSA were significantly more likely to take hypnotics and antidepressants, which are well known to increase the arousal threshold [3]. Patients with REM OSA have more symptoms of insomnia and depression, rather than daytime sleepiness $[5,6]$. Therefore, we assume that many are prescribed hypnotics or antidepressants before a sleep examination. Furthermore, after adjusting for the medication including hypnotics, antidepressants, and antipsychotics, the multivariate logistic regression model showed 
that REM-predominant OSA (adjusted OR, 3.94; 95\% CI, 3.03-5.10; $p<0.001$ ) and REM-isolated OSA (adjusted OR, 16.57 ; 95\% CI, 6.62-41.45) were significantly associated with developing an arousal threshold score of $\geq 2$. These results indicate that pharmacotherapy targets that increase the respiratory arousal threshold might be effective for patients with REM OSA, especially in REM-isolated OSA.

Our results should be interpreted with caution. The method for scoring the respiratory arousal threshold was an indirect estimation. Therefore, our results need to be verified using direct measurements of the respiratory arousal threshold.

In conclusion, compared with patients with non-REM OSA, significantly more patients with REM OSA demonstrated low respiratory arousal thresholds. A low respiratory arousal threshold may be an important endotype that contributes to the pathogenesis of REM OSA, especially in patients with REM-isolated OSA. Further studies are necessary to continue the development of personalized management strategies for patients with REM OSA.

Acknowledgements The authors wish to thank the patients whose clinical records were included in this study and the sleep technicians who manually scored the PSG data, including Yoko Murakami, Momona Arii, Jyunko Hiraki, Takehiro Yamaguchi, Aki Arita, and Masato Imai.

Authors' contributions Conceptualization: Tetsuro Hoshino. Methodology: Tetsuro Hoshino. Validation: Reiko Hori, Mamiko Mano, Atsuhiko Nomura, Noriyuki Konishi, Masayo Baku, and Wojciech Kuczynski. Formal analysis: Tetsuro Hoshino and Kenta Murotani. Investigation: Tetsuro Hoshino and Yoshitomo Nishio. Resources: Chihiro Kato. Writing, original draft preparation: Tetsuro Hoshino. Writing, review and editing: Tetsuro Hoshino. Supervision: Toshiaki Shiomi. Project administration: Ryujiro Sasanabe. All authors have read and agreed to the published version of the manuscript.

\section{Declarations}

Ethics approval The study was approved by the Institutional Review Board (IRB) of Aichi Medical University Hospital and was conducted in accordance with the amended Declaration of Helsinki (approval number 2020-088). The IRB granted a waiver of the patients' informed consent because of the noninvasive and retrospective nature of the study. However, we published an outline of this study plan for public viewing on the Aichi Medical University website.

Conflict of interest The authors declare no competing interests.

Open Access This article is licensed under a Creative Commons Attribution 4.0 International License, which permits use, sharing, adaptation, distribution and reproduction in any medium or format, as long as you give appropriate credit to the original author(s) and the source, provide a link to the Creative Commons licence, and indicate if changes were made. The images or other third party material in this article are included in the article's Creative Commons licence, unless indicated otherwise in a credit line to the material. If material is not included in the article's Creative Commons licence and your intended use is not permitted by statutory regulation or exceeds the permitted use, you will need to obtain permission directly from the copyright holder. To view a copy of this licence, visit http://creativecommons.org/licenses/by/4.0/.

\section{References}

1. Eckert DJ (2016) Phenotypic approaches to obstructive sleep apnoea-new pathways for targeted therapy. Sleep Med Rev 37:45-59. https://doi.org/10.1016/j.smrv.2016.12.003

2. Mano M, Hoshino T, Sasanabe R, Murotani K, Nomura A, Hori R, Konishi N, Baku M, Shiomi T (2019) Impact of gender and age on rapid eye movement-related obstructive sleep apnea: a clinical study of 3234 Japanese OSA patients. Int J Environ Res Public Health 16:E1068. https://doi.org/10.3390/ijerph16061068

3. Taranto-Montemurro L, Messineo L, Wellman A (2019) Targeting endotypic traits with medications for the pharmacological treatment of obstructive sleep apnea. A review of the current literature. J Clin Med 8(11):1846. https://doi.org/10.3390/jcm8111846

4. Varga AW, Mokhlesi B (2019) REM obstructive sleep apnea: risk for adverse health outcomes and novel treatments. Sleep Breath 23:413-423. https://doi.org/10.1007/s11325-018-1727-2

5. Geckil AA, Ermis H (2019) The relationship between anxiety, depression, daytime sleepiness in the REM-related mild OSAS and the NREM-related mild OSAS. Sleep Breath 24:1-5. https:// doi.org/10.1007/s11325-019-01838-y

6. Hoshino T, Sasanabe R, Murotani K, Hori R, Mano M, Nomura A, Konishi N, Baku M, Arita A, Kuczynski W, Shiomi T (2020) Insomnia as a symptom of rapid eye movement-related obstructive sleep apnea. J Clin Med 9:1821. https://doi.org/10.3390/jcm90 61821

7. Gleeson K, Zwilllch CW, White DP (1990) The influence of increasing ventilatory effort on arousal from Sleep1-3. Am Rev Respir Dis 142:295-300. https://doi.org/10.1164/ajrccm/142.2. 295

8. Messineo L, Eckert DJ, Lim R, Chiang A, Azarbarzin A, Carter SG, Carberry JC (2020) Zolpidem increases sleep efficiency and the respiratory arousal threshold without changing sleep apnoea severity and pharyngeal muscle activity. J Physiol 598:46814692. https://doi.org/10.1113/JP280173

9. Edwards BA, Eckert DJ, McSharry DG et al (2014) Clinical predictors of the respiratory arousal threshold in patients with obstructive sleep apnea. Am J Respir Crit Care Med 190(11):1293-1300. https://doi.org/10.1164/rccm.201404-0718OC

10. Berry R, Brooks R, Gamaldo C, Harding S, Marcus C, Vaughn B, Tangredi MM (2012) The AASM manual for the scoring of sleep and associated events: rules, terminology and technical specifications, Version 2.0. Darien, Illinois: American Academy of Sleep Medicine.

11. American Academy of Sleep Medicine (2005) International classification of sleep disorders. Diagnostic and coding manual (ICSD2), 2nd ed.; American Academy of Sleep Medicine: Westchester, IL.

12. El-Solh AA, Lawson Y, Wilding GE (2020) Impact of low arousal threshold on treatment of obstructive sleep apnea in patients with post-traumatic stress disorder. Sleep Breath. https://doi.org/10. 1007/s11325-020-02106-0

13. Zinchuk A, Edwards BA, Jeon S, Koo BB, Concato J, Sands S, Wellman A, Yaggi HK (2018) Prevalence, associated clinical features, and impact on continuous positive airway pressure use of a low respiratory arousal threshold among male United States veterans with obstructive sleep apnea. J Clin Sleep Med 14(5):809-817. https://doi.org/10.5664/jcsm.7112

14. Hoshino T, Sasanabe R, Tanigawa T, Murotani K, Arimoto M, Ueda H, Shiomi T (2018) Effect of rapid eye movement related obstructive sleep apnea on adherence to continuous positive 
airway pressure. J Int Med Res 46:2238-2248. https://doi.org/10. $1177 / 0300060518758583$

15. Almeneessier AS, Almousa Y, Hammad O, Olaish AH, ALAnbay ET, BaHammam AS (2017) Long-term adherence to continuous positive airway pressure in patients with rapid eye movement only obstructive sleep apnea: a prospective cohort study. J Thorac Dis 9:3755-3765. https://doi.org/10.21037/jtd.2017.09.57
Publisher's note Springer Nature remains neutral with regard to jurisdictional claims in published maps and institutional affiliations. 